\title{
L'EUROPE MAÎTRISE-T-ELLE SES ACCORDS DE LIBRE-ÉCHANGE AGRICOLE ?
}

\author{
Tancrède Voituriez \\ in Sébastien Abis et al., Le Déméter 2020
}

IRIS éditions | « Hors collection »

2020 | pages 81 à 99

ISBN 0011662116

Article disponible en ligne à l'adresse :

https://www.cairn.info/le-demeter---page-81.htm

Distribution électronique Cairn.info pour IRIS éditions.

(C) IRIS éditions. Tous droits réservés pour tous pays.

La reproduction ou représentation de cet article, notamment par photocopie, n'est autorisée que dans les limites des conditions générales d'utilisation du site ou, le cas échéant, des conditions générales de la licence souscrite par votre établissement. Toute autre reproduction ou représentation, en tout ou partie, sous quelque forme et de quelque manière que ce soit, est interdite sauf accord préalable et écrit de l'éditeur, en dehors des cas prévus par la législation en vigueur en France. Il est précisé que son stockage dans une base de données est également interdit. 


\title{
L'EUROPE MAÎTRISE-T-ELLE SES ACCORDS DE LIBRE-ÉCHANGE AGRICOLE?
}

\author{
Tancrède Voituriez
}

Chercheur senior en gouvernance du développement durable à l'Institut du développement durable et des relations internationales (IDDRI) et chercheur au sein de l'Organisme français de recherche agronomique et de coopération internationale pour le développement durable des régions tropicales et méditerranéennes (CIRAD) au Nigeria.

ongtemps prudente et mesurée à l'égard des accords de libre-échange bilatéraux (ALE), I'Union européenne (UE) est aujourd'hui l'un de leurs promoteurs les plus actifs. Occupée pendant vingt ans à agrandir son marché intérieur, elle se tourne désormais vers l'extérieur. Sa faveur pour le bilatéralisme commercial s'incarne dans le contenu même des accords, qui, limités dans un premier temps à l'échange de biens et services, touchent aujourd'hui un large éventail de dispositions et de politiques publiques, incluant l'investissement, la propriété intellectuelle, les règles d'accès au marché public et les normes sanitaires et environnementales. L'élargissement géographique et thématique des ALE n'est cependant pas sans risques. Le premier pour l'UE est que leurs conséquences politiques et sociales, in fine, lui échappent, et qu'un instrument vanté pour ses multiples bienfaits - mais encore imprécis ou allusif sur nombre de sujets sensibles au cœur des choix collectifs des citoyens - n'en vienne à subordonner l'intérêt général à celui de quelques-uns.

Ce chapitre dresse un état des lieux de l'engagement de l'UE envers le bilatéralisme commercial. II sonde les raisons de ce choix, et dégage les controverses essentielles entourant les gains, les coûts et les risques associés aux ALE dits de «nouvelle génération », pour enfin explorer quelques pistes et principes de réforme des ALE à même de réconcilier l'agenda commercial de l'Union avec les préférences collectives de ses citoyens, en particulier en matière d'environnement et de santé. L'absence de vision commune concernant la finalité de la politique commerciale de l'UE n'a d'égale que celle touchant aux objectifs de long terme de la politique agricole. De l'intérêt, donc, d'éclaircir la vision de l'agriculture de demain, de rétablir l'ordre des priorités selon lequel la libéralisation est un moyen au service de celle-ci, et non une fin que personne ne comprend plus. 


\section{L'UE ET LES ACCORDS DE LIBRE-ÉCHANGE BILATÉRAUX, UNE CONVERTIE TARDIVE MAIS CONVAINCUE}

\section{L'essor des ALE après la création de l'OMC}

À peu près inexistants dans la période du GATT (Accord général sur les tarifs douaniers et le commerce, 1947-1995) courant depuis l'immédiat après-guerre jusqu'à la création de l'Organisation mondiale du commerce (OMC) en 1994, les ALE sont depuis vingt ans un des plus puissants moteurs, sinon le plus puissant, de la mondialisation. Leur développement semble irrésistible et si des résistances farouches freinent aujourd'hui leur accroissement, celles-ci restent bien moindres que celles tenant à l'arrêt complet des négociations de libéralisation multilatérale de l'agriculture à l'OMC depuis dix ans.

En septembre 2019, 291 accords commerciaux régionaux de libre-échange étaient actifs dans toutes les régions du monde. Environ la moitié ne couvre que les biens, une autre moitié couvre les biens et services, et seul un ALE couvre uniquement les services. Comme pour sa dimension multilatérale, le commerce bilatéral est encadré par des règles conçues avant tout pour l'échange de produits. Sous cet aspect, il est le miroir inverse de nos économies - à tout le moins celle des pays riches. Le produit intérieur brut (PIB) des pays de l'Organisation de coopération et de développement économiques (OCDE) est composé à $75 \%$ de services et $25 \%$ de biens, en moyenne. Le commerce international, commerce bilatéral inclus, est l'exact inverse, avec $75 \%$ d'échanges de biens et tout juste $25 \%$ de services.

L'Espace économique européen (EEE) - incluant I'UE et l'Association européenne de libre-échange (AELE, incluant I'Islande, le Liechtenstein, la Norvège et la Suisse) - est aujourd'hui partie prenante de 67 ALE. Cette liste contient cependant quelques doublons, l'UE et l'AELE signant dans un certain nombre de cas les mêmes accords à quelques mois près. Si l'on s'en tient à l'Europe politique, et donc à l'UE, le nombre d'ALE en vigueur avec des pays extérieurs à l'EEE s'établit, en septembre 2019, à 37. Rapporté à son PIB, ce chiffre n'est en réalité pas très élevé quand on le compare à celui d'autres blocs régionaux. Selon les données de l'OMC, le champion des ALE reste l'Asie orientale (86), suivie par l'Amérique du Sud (6I), la Communauté des États indépendants (46), l'Amérique du Nord (42), puis l'Amérique centrale (40). L'UE devance tout juste l'Afrique (34). En queue de classement, on trouve le Moyen-Orient, l'Océanie, et l'Asie occidentale (entre 22 et 29) puis les Caraïbes (neuf). II ressort de cette arithmétique que, contrairement à la présentation qu'en fait l'OMC, où il apparaît que l'Europe est le champion toute catégorie des ALE - avec le chiffre de 99 accords en vigueur depuis les tout premiers temps de la construction européenne -, l'UE est une puissance commerciale dont l'engagement bilatéral reste minoritaire au regard des efforts consentis pour développer le commerce à l'intérieur de l'espace européen. Cet état de fait n'est pas contra- 
dictoire avec le fait que l'UE se projette aujourd'hui comme un des acteurs les plus dynamiques et engagés dans la négociation d'ALE.

La nature des ALE signés puis mis en œuvre a évolué avec le temps. Durant la période du GATT, l'essentiel des quelques ALE était de nature politique : construction de la Communauté économique européenne (CEE) puis de l'UE, intégration commerciale des pays de l'ancien bloc de l'Est après l'effondrement de l'Union soviétique. Les créations de zones de libre-échange telles que l'Association des nations de l'Asie du Sud-Est (Asean, mise en œuvre en 1993), l'Accord de libreéchange nord-américain (Alena, 1994)' et l'EEE (1994) en fin de période d'application du GATT marquent la transition vers des accords de dimension exclusivement économique, dans une parenthèse de fin de l'histoire et d'hégémonie de la démocratie libérale établissant le commerce international comme un des moyens les plus sûrs de faire «tenir le monde ensemble», pour reprendre l'expression du politiste John Gerard Ruggie 2 . Intermède d'un peu plus de dix ans finalement clos par les attentats du I I septembre 200 I et l'excédent commercial chinois.

\section{Pourquoi l'UE s'est-elle convertie aux ALE?}

L'histoire de la conversion de I'UE aux ALE - hors ALE intraeuropéens - est indissociable d'une autre histoire, celle de la construction européenne. Elle est aussi intimement liée à l'identité de ses commissaires au Commerce, dont la passion pour le multilatéralisme a connu des fortunes diverses à mesure que les négociations à l'OMC s'enlisaient et que montaient les chants de sirènes des ALE. À la création de l'OMC, en décembre 1994, les ALE étaient conçus comme une tolérance, une dérogation nécessaire dans un corpus doctrinaire tout entier construit sur le principe de non-discrimination entre pays partenaires. Un ALE constitue en effet une violation caractérisée de ce principe, puisqu'il autorise les États parties à un accord bilatéral ou régional d'échanger entre eux sur une base tarifaire plus favorable. Un produit importé dans un pays A, membre d'un ALE, sera alors frappé de deux droits de douane différents selon sa provenance : droit de douane nul s'il vient du pays $B$ également membre de l'ALE, et droit de douane de la nation la plus favorisée (NPF) s'il provient d'un pays membre de l'OMC, non partie à l'ALE.

Après des controverses et la production de données empiriques disputant la nature des contributions des ALE au multilatéralisme commercial, l'OMC conserve l'idée que sous certaines conditions, énoncées dans l'article XXIV du GATT, les ALE peuvent être un composant fondateur (building block) plutôt qu'une pierre d'achoppement (stumbling block) de la libéralisation commerciale multilatérale. Ils sont, dans l'esprit de l'article, un moyen, et non une fin - celle-ci restant l'accroissement du commerce et des revenus par la voie multilatérale.

\footnotetext{
I. Remplacé en 2019 par l'Accord États-Unis-Mexique-Canada (AEUMC).

2. John Gerard Ruggie, «What Makes the World Hang Together? Neo-Utilitarianism and the Social Constructivist Challenge», International Organization, vol. 52, n 4, 1998.
} 


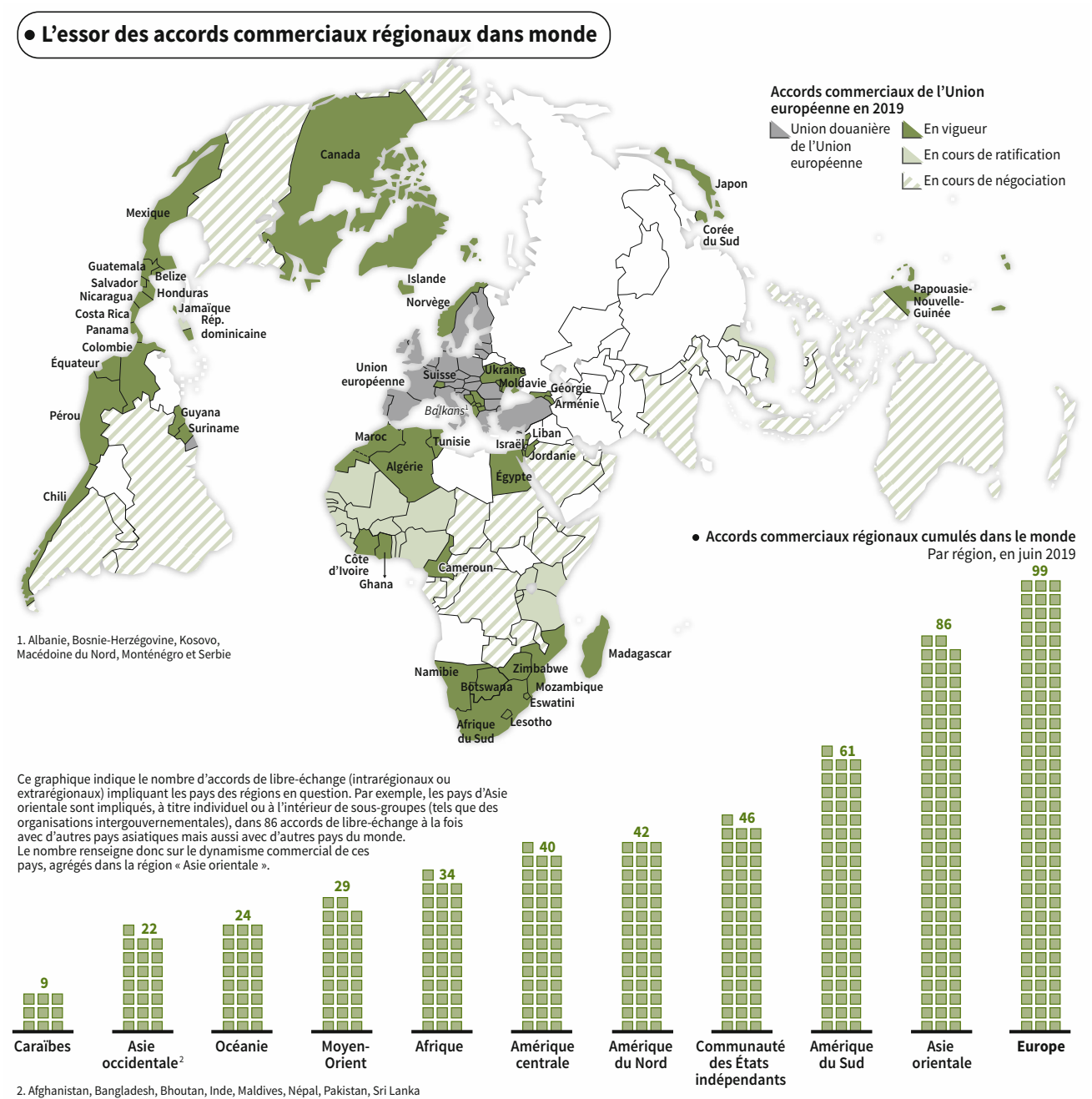

- Accords commerciaux régionaux dans le monde en septembre 2019

Nombre

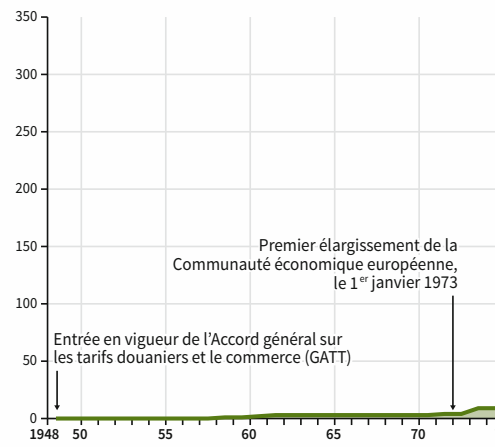

Sources : Commission européenne, 2019; OMC, 2019

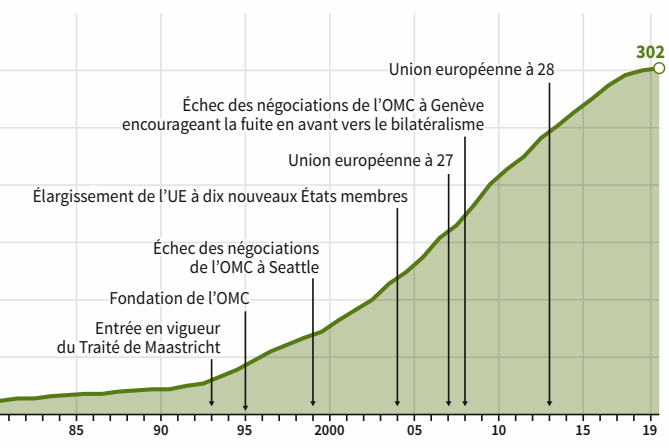

Réalisation cartographique : Laura Margueritte @ DEMETER / Le Déméter 2020 
L'UE épousa cette idée de 1995 à 2004, dans une période où son agenda politique ne lui permettait pas de se lancer dans des négociations commerciales bilatérales. Son calendrier commercial bilatéral était alors dominé pour l'essentiel par l'élargissement à 10 nouveaux États, portant le nombre de ses membres à 25 pays en mai 2004. La transformation de la relation avec les pays d'Afrique, Caraïbes, Pacifique (ACP), et la signature du programme d'accords de partenariat économique (APE) réciproque avec ceux-ci sont l'exception qui confirme la règle de priorité donnée à l'élargissement.

En plus du peu de temps matériel laissé à la négociation d'accords commerciaux bilatéraux, l'élargissement de l'UE de 15 à 25 pays en tout juste dix ans a eu cet effet de renforcer le besoin des institutions européennes de se construire une existence morale. Les entretiens menés à l'époque au sein de la direction générale (DG) du Commerce montrent le souci de la Commission de ne pas apparaître comme une entité bureaucratique, sans vision et sans âme, et de répondre aux attentes d'une société civile dont on découvre depuis la conférence ministérielle de I'OMC à Seattle (1999) la virulence, l'opiniâtreté et la capacité d'adhésion et de conviction auprès de l'opinion. La mise en place systématique d'études d'impact sur le développement durable (trade sustainability impact assessment) et les rencontres régulières à Bruxelles avec les membres de la société civile accréditée, témoignent de cet effort de I'UE, constant durant le mandat de Pascal Lamy (1999-2004), de faire de la DG Commerce une enceinte produisant à la fois des accords et du sens - celui selon lequel le propre du commerce est de porter à la paix, clef de voûte et raison d'être de la construction européenne.

Une dernière raison derrière l'étroite adhésion de I'UE au projet multilatéral de l'OMC jusqu'à 2004 tient donc à son commissaire, P. Lamy. Européen convaincu, passé par le cabinet de Jacques Delors lorsque celui-ci était président de la Commission, il n'a eu de cesse de vouloir donner du sens au commerce. Contrairement à ses successeurs, P. Lamy privilégiait sans ambiguiité le multilatéralisme au commerce - pour ce que le multilatéralisme signifiait pour l'Europe alors, puissance soft et non hard. Ce fut probablement moins le cas des commissaires suivants, pour des raisons qui relèvent sans doute autant de parcours et de convictions personnelles que de l'impossibilité, chaque année plus clairement manifeste, de conclure les négociations multilatérales à l'OMC après qu'en juillet 2008 un «presque accord» fut, in fine, rejeté.

\section{L'état du calendrier et des négociations de l'UE}

L'UE a devant elle trois chantiers de négociations commerciales. L'accord commercial avec le Royaume-Uni, la réforme de l'OMC et les accords bilatéraux. Nous insisterons dans ce chapitre sur ces derniers. Le calendrier bilatéral de l'UE en matière commerciale est rythmé par la négociation et la signature de trois types d'accords: les unions douanières (libre commerce bilatéral à l'abri d'un tarif extérieur commun); les accords d'association, de stabilisation 
et d'association (ASA) et les accords de libre-échange (ALE) et de partenariat économique (APE), qui visent à réduire les barrières au commerce entre participants - sans nécessairement les supprimer; les accords de coopération et de partenariat, qui sont des accords-cadres dépourvus de dispositions touchant aux barrières douanières. Nous nous intéressons ici à la deuxième catégorie, qui réunit l'essentiel des initiatives en cours de l'UE (voir carte).

Les ALE et les APE en cours de négociation par I'UE concernent tous les continents et sont d'ampleur et d'ambition diverses. Ainsi l'accord entre I'Union et les États-Unis - qui vise pour l'essentiel à la réduction de droits de douane sur certains produits industriels - de 2018 peut-il être qualifié de restreint, quand le CETA - accord économique et commercial global entre I'UE et le Canada, partiellement en vigueur depuis septembre 2017 - est décrit comme un accord complet (comprehensive), réunissant dans un même cadre les dispositions touchant au commerce des biens, des services, la propriété intellectuelle - appellations protégées incluses -, les marchés publics et l'investissement. Pour cette raison, ce dernier type d'accord est qualifié d'accord de nouvelle génération - la première se concentrait sur la réduction des obstacles tarifaires au commerce des biens et éventuellement des services, ils tendent, désormais, vers une convergence ou une harmonisation des normes, qu'elles soient sanitaires, sociales, techniques ou environnementales.

L'expression «accord de nouvelle génération » a commencé à être employée au sein de l'UE à partir de la négociation de l'accord de libre-échange avec la Corée du Sud, en 20I I, mais a en réalité été lancée à la fin des années 2000. Ils concernent aujourd'hui la plupart des accords commerciaux négociés par I'UE : il en est ainsi de l'accord d'association avec l'Ukraine, entré partiellement en vigueur depuis janvier 2016, du CETA, mais aussi de l'accord avec le Japon (JEFTA), en vigueur depuis février 2019 (voir l'encadré à ce sujet en fin de chapitre). D'autres accords, conclus à la fin des années 2000, suivent la même logique : Pérou, Colombie, Équateur, Amérique centrale (Costa Rica, Salvador, Guatemala, Honduras, Nicaragua et Panama). Des accords avec Singapour et le Viêtnam sont en cours de ratification. D'autres sont actuellement négociés ou étendus afin d'être «complets», comme avec le Marché commun du Sud (Mercosur), le Mexique, le Chili, l'Australie, la Nouvelle-Zélande, l'Indonésie, les Philippines et la Tunisie. Fait notable, ni I'Inde, en dépit d'une tentative infructueuse entre 2007 et 2013, ni la Chine ne sont engagées dans des négociations d'ALE avec I'UE!.

I. La Chine et l'UE sont en cours de négociation d'un accord restreint à l'investissement depuis novembre 2013. 


\section{POURQUOI LES ALE SONT-ILS SI DIFFICILES À NÉGOCIER?}

\section{Les gains attendus des ALE pour l'UE}

Les gains attendus des ALE de première génération, qui se concentraient sur les produits plus que sur les services, et visaient pour l'essentiel à réduire les barrières douanières sans trop se préoccuper des normes et des arsenaux règlementaires encadrant la production, découlent de l'économie la plus basique. Toutes choses égales par ailleurs, un droit de douane augmente le prix des produits importés sur le marché intérieur, et de cette augmentation découlent des effets contradictoires chez les consommateurs et les producteurs - effets qui ne se compensent pas : les consommateurs dépensent plus et les producteurs accroissent leurs profits. Par rapport à une situation de libre-échange, la perte des premiers est supérieure aux gains des seconds, de sorte que l'effet global d'un droit de douane sur l'économie est jugé négatif - les recettes douanières engrangées par le gouvernement ne modifiant pas le signe du résultat global de l'addition des pertes du consommateur et des gains des producteurs et de l'État. L'intérêt égoïste d'un pays serait donc, en théorie, de renoncer à un protectionnisme complet. Évidemment, la théorie est critiquable : elle ne tient pas compte de la taille du pays, qui peut influencer le prix mondial et tirer un bénéfice stratégique de la baisse des cours provoqués par ses droits de douane; et elle est portée par un raisonnement statique, ignorant du temps et de son corollaire, le risque, deux notions fondamentales en agriculture. Reste que les équipes de négociation de la Commission européenne sont plus familières de cette économie «standard» que des subtilités de l'économie rurale, l'agriculture étant pour elle un secteur comme les autres. La baisse des barrières douanières accroît le bien-être d'un pays - disons pour simplifier, son revenu - en statique comparative', la question se posant au gouvernement restant celle de compenser les perdants.

Les gains d'une libéralisation «complète», pour reprendre l'adjectif de la Commission, sont plus subtils et délicats à mesurer, à tout le moins avec les modèles et les schémas de pensée retenus pour les accords de première génération. Tout d'abord, alors que la théorie néoclassique du commerce international voit des gains systématiques à l'échange, elle demeure bien plus circonspecte pour tout ce qui a trait aux mesures non tarifaires touchant à la production, telles que les normes, les subventions ou la protection de la propriété intellectuelle. Bon nombre de ces mesures produisent du bien public, en corrigeant des défaillances du marché - ici l'absence d'information crédible, vérifiée et partagée, là la destruction de paysages ou de la biodiversité. Pour cette économie dominante, un droit de douane est une nuisance, même lorsque le marché intérieur est grevé de «défaillances de mar-

I. «En statique comparative, on détermine à un instant donné les conditions de l'équilibre sur une courte période, pour rechercher les raisons qui font que les grandeurs économiques se fixent à un niveau inférieur à celui qui assurerait le pleinemploi, par comparaison avec les conditions de réalisation de l'équilibre correspondant à celui-ci.», Encyclopédie Universalis. 
ché »' . En revanche, pour cette même économie, des mesures intérieures ciblant les défaillances en question sont une absolue nécessité. Hors de question, donc, de s'en débarrasser.Tout le jeu consiste alors à les rapprocher entre pays, afin de faciliter les échanges et d'accroître les gains nets attendus de la suppression des tarifs douaniers.

La mesure des gains escomptés d'une libéralisation complète est un autre problème. Les mesures concernées (modification ou convergence de normes ou règles applicables à la sécurité alimentaire et sanitaire, ainsi qu'à l'environnement, décisions affectant la propriété intellectuelle, les travailleurs détachés, la reconnaissance réciproque des diplômes, etc.) sont hétérogènes par nature, et plus difficiles à mettre en équation qu'un droit de douane qui affecte directement les prix. Les modèles utilisés pour simuler les conséquences économiques des ALE sont pour l'essentiel des modèles d'équilibre général calculable, parfois assortis de modèles d'équilibre partiel s'intéressant à un produit ou à un secteur particulier. La clef de voûte de ces modèles est le prix : tous les comportements sont mus par une certaine sensibilité à celui-ci. Le défi donc est de traduire l'effet des changements de normes ou de règlements en changements de prix - lesquels, en cascade, provoqueront des variations dans les niveaux de consommation et de profit par produit. II y a inévitablement une part d'arbitraire dans cette mise en équation, qui relève davantage du bricolage que de l'application sourcilleuse d'une théorie. Ces réserves posées, il est possible de s'arrêter sur les résultats et controverses ayant entouré les simulations des conséquences du CETA, emblématique de ces accords de nouvelle génération négociés par l'UE.

\section{Libéraliser pour quoi faire? Les contradictions de l'approche européenne}

Le CETA a été signé avec le Canada le 30 octobre 2016, ratifié par le Parlement européen le 15 février 2017 et est entré en vigueur de façon provisoire le 21 septembre 2017. Cette dernière ne sera pleinement effective qu'après l'achèvement de toutes les procédures nationales de ratification. L'UE est le deuxième fournisseur de biens du Canada derrière les États-Unis, et le Canada est le douzième marché pour les exportateurs de l'UE à 28 , hors commerce intracommunautaire. Les produits agricoles et alimentaires - céréales, oléoprotéagineux, viandes et boissons en tête - représentent près de $10 \%$ des échanges en valeur avec le Canada, avec qui la balance commerciale agricole et alimentaire est actuellement excédentaire, essentiellement grâce au poste des boissons. A contrario, le déficit se creuse en céréales et oléagineux et, dans une moindre mesure, en préparation de viandes et poissons².

Au sein de I'UE, la France est le plus important exportateur de produits alimentaires transformés vers le Canada, lesquels représentent $24 \%$ des exportations

\footnotetext{
I. Jagdish N. Bhagwati et Virudhunagar K. Ramaswami, «Domestic Distortions, Tariffs, and the Theory of Optimum Subsidy», Journal of Political Economy, vol. 76, n 6, 1969.

2. Aurélie Trouvé, Baptiste Buczinski, Philippe Chotteau, Estelle Antoine, Hervé Marouby, Michel Rieu et Jan-PeterVan Ferneij, Analyse de l'accord de libre-échange entre l'Union européenne et le Canada (AECG/CETA) dans le secteur des produits animaux, Rapport AgroParisTech, Idele, IFIP et Université de Laval (Québec), 2017.
} 
européennes, notamment dans les secteurs des vins et spiritueux, du fromage et de l'eau minérale. Le Canada est, pour sa part, le quatorzième fournisseur extracommunautaire de I'UE, dont les ventes annuelles de denrées alimentaires et boissons au sein du marché unique ont atteint 1,7 milliard d'euros en 2017. Le CETA organise le démantèlement de 93,8\% des lignes tarifaires à l'entrée de l'UE et de 91,7\% à l'entrée du Canada. Toutefois, certains produits qualifiés de «sensibles» ont fait l'objet d'un traitement différencié, ainsi des viandes bovine et porcine et de certains produits sucrés (contingentés), ou de la volaille de chair, exclue de la libéralisation!.

Les arguments de la Commission européenne mettent en avant les opportunités de commerce et d'investissements pour les acteurs économiques des deux pays partageant les mêmes valeurs et les mêmes intérêts ${ }^{2}$. Deux chiffres figurent en tête du rapport : la hausse des échanges bilatéraux de $8 \%$, survenant pour l'essentiel la première année de mise en œuvre, et une hausse de PIB de l'UE d'environ 2 milliards d'euros par an - soit 0,01\% du PIB européen. Un rapport antérieur, reposant sur des hypothèses d'une libéralisation plus large et de gains d'innovations plus substantiels, prédisait une hausse maximale du PIB européen à long terme de $0,02 \%$ à $0,03 \%$ par $a n^{3}$. Des résultats faibles, qui n'ont en réalité rien d'étonnant: les gains - mesurés avec les réserves précédemment évoquées - sont proportionnels au niveau général de protection. Or les économies canadiennes et européennes sont aujourd'hui parmi les plus ouvertes au monde, et c'est ironiquement cette ouverture héritée du passé qui réduit les perspectives de gain d'un surcroît d'ouverture demain.

La contribution des ALE à la croissance européenne est de l'ordre donc du centième de point de PIB et se situe, de ce fait, dans la marge d'erreur des modèles. Le gain économique, à l'échelle des nations européennes, est modeste, quand les effets de répartition entre secteurs et ménages peuvent, eux, être importants. L'économiste états-unien Dani Rodrik ${ }^{4}$ démontrait d'ailleurs que l'effet marginal de la libéralisation du commerce sur la croissance diminue au cours du temps, tandis que son effet sur la distribution des revenus est croissant. À mesure qu'une économie s'ouvre au commerce, les gains de croissance tirés d'une poursuite de la libéralisation baissent, tandis que les effets de répartition augmentent. Pour le dire autrement, la libéralisation d'une économie déjà amplement ouverte peut faire perdre 99 à $X$ et fait gagner 100 à Y, pour un gain net de I. La libéralisation d'une économie ouverte, où ne subsistent plus que de rares et tenaces protections, redistribue les gains et les pertes entre secteurs et entre ménages bien davantage qu'elle ne crée de richesse de manière agrégée. C'est bien le contexte dans lequel se trouvent le Canada et I'UE. Le droit de douane moyen - ad valorem appliqué et équivalent

\footnotetext{
I. Commission européenne, Direction générale du Commerce, The Economic Impact of the Comprehensive Economic and Trade Agreement (CETA), Luxembourg, Office des publications de l'Union européenne, 2017.

2. Ibid.

3. Colin Kirkpatrick, Selim Raihan, Adam Bleser, Dan Prud'homme, Karel Mayrand, Jean Frederic Morin, Hector Pollitt, Leonith Hinojosa et Michael Williams, «Trade sustainability impact assessment (SIA) on the comprehensive economic and trade agreement (CETA) between the EU and Canada: Final report», MPRA Paper, $n^{\circ} 288$ I2, University Library of Munich, 201 I. 4. Dani Rodrik, «The Rush to Free Trade in the Developing World: Why So Late? Why Now? Will it Last?», NBER Working Paper, n³947, National Bureau of Economic Research (NBER), 1992.
} 
ad valorem des droits spécifiques - opposé par le Canada aux exportations européennes est de $4,6 \%$, et celui imposé par l'Europe aux produits de son partenaire est de 3,3\%. Les gains «hors droits de douane», liés au rapprochement des normes et des arsenaux règlementaires, ne modifient pas le résultat.

Les simulations de libéralisation dans le secteur de l'agriculture en donnent un exemple probant. L'agriculture - et particulièrement au Canada, avec un droit moyen de 7,3\% contre 11,6\% en Europe - est bien plus protégée des deux côtés que l'industrie - respectivement dans un rapport de I à 4 et de I à 6'. La Commission met en avant les gains économiques², en particulier pour les fromages - doublement du contingent en franchise de droits de douane -, les vins et spiritueux - élimination d'«importantes barrières»-, les chocolats, confiseries, pains, pâtisseries et biscuits - droits de douane à zéro. Elle insiste également sur le fait que 143 indications géographiques (IG) seront protégées (voir l'encadré en fin de chapitre); se prévaut d'avoir protégé les lignes de produits les plus sensibles, avec des ouvertures de contingents tarifaires finalement limités en volumes à des niveaux très faibles par rapport à la production de l'UE; et se flatte d'avoir tenu bon, du moins pour l'instant, sur la garantie d'interdiction des hormones ou de la ractopamine dans les produits d'importation.

Des évaluations - indépendantes des services de la Commission - nuancent ces résultats. Ainsi le «rapport Schubert» ${ }^{3}$ rappelle que pour la viande bovine, le CETA prévoit une augmentation des quotas d'importation en franchise de droits à l'entrée de l'UE à 67950 tonnes équivalent carcasse (téc) en 2022. La filière pourrait être affectée si l'accord donnait lieu à l'entrée sur le marché de morceaux de haute qualité à moindre coût, déstabilisant l'équilibre économique entre les différents morceaux des carcasses. Les exportateurs canadiens, «très performants dans leur capacité à diversifier leurs débouchés», se verront donc offrir de nouvelles opportunités pour développer une production porcine sans ractopamine et bovine sans hormone, avec un contingent offert promis à augmentation au cours du temps 4 . En outre, «si l'on considère que le CETA a valeur de modèle pour les accords régionaux futurs, il sera difficile de ne pas concéder aux nouveaux partenaires des contingents d'importation de viande plus élevés que ceux qui existent actuellement, ce qui pourra changer notablement l'échelle des problèmes $\rangle^{5}$. Comme le rappellent pragmatiquement Geoffrey Bannister et Kamau Thugge, «même les réformes des politiques commerciales les mieux conçues créent des perdants $\rangle^{6}$. À faire la réclame des ALE, dont les finalités économiques restent peu substantielles pour le vulgum

\footnotetext{
I. Soulignons, avec le rapport Schubert, que la France, compte tenu de sa structure d'exportations agricoles, fait face à des droits de douane plus élevés que la moyenne européenne à l'entrée au Canada (29\%).

2. Commission européenne, «Le CETA et l'agriculture. Les bénéfices pour l'agriculture de l'UE», Fiche d'information CETA 2 sur 7,2017.

3. Jean-Luc Angot, Geneviève Bastid Burdeau, Christophe Bellmann, Sophie Devienne, Lionel Fontagné, Roger Genet, Géraud Guibert, Sabrina Robert-Cuendet et Katheline Schubert, «L'impact de l'Accord Économique et Commercial Global entre I'Union européenne et le Canada (AECG/CETA) sur l'environnement, le climat et la santé. Rapport au Premier Ministre», 2017.

4. Aurélie Trouvé et al., op.cit.

5. Jean-Luc Angot et al., op.cit.

6. Geoffrey J. Bannister et Kamau Thugge, «International Trade and Poverty Alleviation», IMF Working Paper, $n^{\circ} 01 / 54$, Fonds monétaire international (FMI), 2001.
} 
pecus, la Commission oublie de traiter la totalité du problème, et de faire sa juste part à la compensation des perdants, aujourd'hui et demain.

\section{La singularité du (des) modèle(s) agricole(s) européen(s) est-elle résiliente au libre-échange?}

II faut cependant rendre justice à la Commission européenne pour ses efforts de prise en compte de bon nombre d'objections soulevées par la société civile et divers experts rassemblés dans le cadre d'une ample consultation publique, depuis la mise en suspens de la négociation du Partenariat transatlantique de commerce et d'investissement (TTIP) avec les États-Unis. Conservant l'exemple du CETA, «vitrine» de l'UE en matière d'accord de nouvelle génération, on notera que le mécanisme de règlement des différends entre États et investisseurs a été substantiellement amélioré au regard de ce qu'il était dans les ALE ou projets d'ALE antérieurs. Les tribunaux arbitraux privés sont remplacés par une cour permanente - première instance et appel - et les juges y sont nommés par les États. Les procédures seront transparentes, les auditions publiques, les observations disponibles en ligne et les parties tierces ayant un intérêt au différend bénéficieront d'un droit d'intervention (amicus curiae). Les investisseurs ne pourront saisir cette juridiction que dans des cas précis d'expropriation, sans indemnisation ou déni de justice. Le droit des États de règlementer dans l'intérêt général, notamment en matière de santé, de sécurité ou d'environnement est, au contraire, inscrit de manière explicite dans le texte de l'accord'.

C'est ainsi que les normes sanitaires au cœur de la particularité européenne - telle l'obligation de production porcine sans ractopamine et bovine sans hormone, qui va au-delà des exigences du Codex Alimentarius ${ }^{2}$ - ont été préservées. Le texte de l'accord rappelle à ce sujet, à de multiples reprises, que les normes environnementales et sanitaires existantes de la France et de l'UE ne doivent pas être mises en danger par le CETA. Les dispositifs actuels liés à l'application dans l'Union du principe de précaution ne sont pas remis en cause.

Les points de vigilance concernent le caractère «vivant» et dynamique des ALE de nouvelle génération, qui comportent un certain nombre d'omissions et de risques relatifs à des imprécisions, lesquelles pourraient être soumises à des interprétations ultérieures éventuellement défavorables à l'intérêt général de l'UE. Les accords de nouvelle génération se doivent d'intégrer les attentes sociétales en matière d'environnement, de santé, d'alimentation et de bien-être animal, ce qui n'est pas le cas pour l'instant ${ }^{3}$. En particulier le CETA est-il muet sur les questions de l'alimentation animale (farines) et de l'administration d'antibiotiques comme

\footnotetext{
I. Voir le texte de l'accord sur le site de la Commission européenne : https://ec.europa.eu/trade/policy/in-focus/ceta/cetachapter-by-chapter

2. «La Commission du Codex Alimentarius [Code alimentaire] est un organe intergouvernemental mixte (FAO/OMS), qui comprend 187 États Membres et une organisation Membre (I'Union européenne). Depuis 1963, elle établit des normes alimentaires internationales harmonisées pour protéger la santé des consommateurs et garantir des pratiques équitables dans le commerce des denrées alimentaires.», selon l'Organisation mondiale de la santé (OMS).

3. Jean-Luc Angot et al., op. cit.
} 
activateurs de croissance, sujet sur lequel le Canada a des pratiques clairement divergentes de l'UE, et pourrait réclamer une révision de ces dernières. Les anabolisants et les antibiotiques utilisés comme facteurs de croissance sont par exemple interdits dans I'UE, alors qu'ils sont autorisés au Canada. Le CETA laisse la possibilité au Canada d'utiliser des facteurs de croissance contenant des antibiotiques, avec toutefois des contraintes de délais d'attente et d'absence de résidus'. De plus, la règlementation canadienne autorise le rinçage et le traitement au chlore de la viande de bœuf et de poulet, interdits en Europe. Très récemment, en juin 20 17, le Canada a d'ailleurs indiqué souhaiter introduire une demande d'utilisation de l'acide citrique et de l'acide péroxyacétique pour la décontamination des carcasses. «Si l'Europe accédait à de telles demandes, cela pourrait à terme remettre en question le modèle de sécurité sanitaire des aliments européen $\rangle^{2}$. De fait, rien n'interdit dans le texte la formulation d'une telle demande par le Canada, non plus que son acceptation par I'UE. Rappelons aussi qu'en vertu de la directive 96/22/CE du Conseil du 29 avril 1996, les substances ayant un double usage, thérapeutique et de promotion de la croissance, que sont les antibiotiques, ne sont pas interdites à l'entrée de l'UE. Que se passerait-il alors si une décision d'interdiction venait à être prise par l'Union dans le cadre de l'accord?

La réponse réside dans l'interprétation que chacun peut faire de la qualité du contrôle démocratique de la mise en œuvre d'un accord au sein de l'UE. Si, comme on l'a vu, des améliorations ont été apportées au mécanisme de règlement des différends, la capacité des ALE à tenir compte au plus près des préférences collectives européennes ne peut être considérée comme acquise à la lecture des rapports produits sur le sujet. Les omissions actuelles laissent un doute sur la volonté de l'Union de conserver intactes ses préférences; et les mécanismes de règlement des différends (système juridictionnel des investissements ou Investment Court System, ICS) et de coopération règlementaire (JCR) n'écartent pas totalement le risque de conflits d'intérêts et de «capture du régulateur» par une interprétation biaisée des «attentes légitimes des entreprises» éventuellement «frustrées» par les décisions des gouvernements au fil du temps. Le risque, en outre, est que les incertitudes sur l'interprétation du CETA créent un effet paralysant sur les gouvernements, lesquels, dans une sorte d'asservissement volontaire, s'abstiendraient de prendre des décisions allant dans le sens de l'intérêt général, par crainte d'être poursuivis par les investisseurs devant la cour et les juges qu'ils auront pourtant nommés.

I. Ibid.
2. Aurélie Trouvé et al., op. cit. 


\section{QUELLES POLITIQUES COMMERCIALES POUR L'UE APRÈS 2020?}

\section{Des ALE, oui, mais pour quoi faire?}

Pour la première fois depuis 1992 et la bifurcation provoquée par la réforme Mac Sharry de la politique agricole commune (PAC), l'Europe doit penser sa politique commerciale et sa politique agricole sans qu'aucune hiérarchie ne s'applique entre les deux. Les grandes réformes de la PAC de 1992 et 2003, qui ont conduit à l'alignement progressif du marché intérieur sur les prix mondiaux et au découplage des aides, ont été conçues dans l'anticipation des revers diplomatiques qui menaçaient I'UE à l'OMC. Ce sont les négociations commerciales, ou leur imminence, qui, dans une grande mesure, ont «fait» la PAC de 1992, puis celle de 2003. La réforme Mac Sharry, quoique largement motivée par des raisons budgétaires propres à l'Europe, est en effet contemporaine de l'accord commercial de Blair House, qui est le décalque anticipé de l'accord de Marrakech à l'OMC. La réforme de 2003 a été promue par le commissaire à l'Agriculture, Franz Fischler, comme le meilleur moyen et la condition sine qua non pour ne pas soulever l'ire des partenaires commerciaux dans la négociation du cycle de Doha à l'OMC, lancée trois ans plus tôt.

Tout cela semble aujourd'hui révolu. Depuis l'été 2008, l'OMC est en panne: l'idée de mettre la libéralisation au service du «développement» s'avère inopérante, et même contreproductive, en l'absence de définition unanime de la liste des pays que le terme recouvre. L'OMC ne sert plus, par anticipation, d'aiguillon aux réformes de la PAC. Le paradoxe est que l'Europe agricole est tout autant enlisée : depuis 2008 et le cacophonique Conseil «Agriculture et pêche» - n'ayant débouché, fait rarissime, sur aucune déclaration ni décision -, l'UE est bien en peine de définir des objectifs consensuels à son agriculture, ce qui s'avère fâcheux pour qui souhaite bâtir une politique agricole commune. Libérée de la contrainte d'une négociation cadenassée sur le schéma de l'accord de Marrakech, elle ne sait que faire de ces nouvelles possibilités.

Sauf coup de théâtre à Genève, siège de l'OMC, la politique commerciale de I'UE dans les prochaines années s'écrira sous la forme de nouveaux ALE, bien davantage que par un nouvel accord multilatéral signé au sein de l'organisation. Une des questions qui se posent est alors de savoir s'ils prendront davantage en compte - que ne l'a fait le CETA - les réserves et les interrogations exprimées à leur sujet. À survendre les ALE, la Commission s'expose à des revers de fortune, comme ce fut le cas avec le TTIP et le CETA dans sa première version. À minimiser l'ampleur des transformations attendues des ALE, elle risque de susciter de la perplexité sur la nécessité de les négocier. Dans un juste milieu, l'honnêteté lui commande d'être transparente sur les gains et les coûts, et bien avant cela, d'être inébranlable sur les finalités que sert la libéralisation commerciale mieux que toute autre politique, dans une économie aujourd'hui parmi les plus intégrées aux marchés mondiaux. 
Une telle clarification met l'Union face à un choix. Celui de poursuivre à tâtons un calendrier de libéralisation commerciale autour duquel elle s'est construite, avec les risques de soulever des protestations toujours plus vives contre le coût social de cette marche en avant dont on ne voit plus où elle mène. Et celui de faire une pause afin d'identifier les finalités communes de nos modèles agricoles, avec le risque de détruire le fragile édifice de la PAC si aucun accord ne pouvait être obtenu. Cette alternative semble préférable : la discussion des fins doit précéder celle des moyens, pour le cas de la PAC comme pour ceux de la libéralisation commerciale et du budget européen.

\section{Réconcilier les ALE avec les préférences collectives européennes}

En 2004, quelques mois avant la fin de son mandat à la DG Commerce, P. Lamy publie en son nom, sans l'aval de la Commission européenne, un texte intitulé «L'émergence des préférences collectives dans le commerce international : implications pour la régulation de la mondialisation $\gg$. II y dresse le constat suivant : l'ouverture internationale est un puissant vecteur de croissance et d'emplois au bénéfice de tous. Mais, aussi bénéfique soit-elle, elle est, dans le même temps, porteuse d'effets déstabilisateurs pour les tissus économiques et sociaux et, potentiellement, pour les choix de société. Les craintes d'une remise en cause des choix sociaux par une OMC qui serait toute-puissante sont au centre de la contestation de la mondialisation et influencent désormais les négociations internationales. Parmi les choix sociaux, il cite la préférence européenne pour du bœuf sans hormone, la précaution en matière de risque, les services publics et les droits sociaux. Le raisonnement sur les risques encourus par une libéralisation sans direction est intéressant, car il est très similaire à celui que l'on peut lire aujourd'hui sur les ALE.

Si la question des effets de l'ouverture sur l'emploi a fait l'objet d'un effort de pédagogie et de mesures d'accompagnement, dit-il en substance, celle d'une possible remise en cause des choix de société n'a pas été considérée de manière suffisamment attentive. Si ces risques étaient avérés, ils seraient d'une nature infiniment plus grave que les effets déstabilisateurs sur l'activité et l'emploi : ils toucheraient au cœur du lien social et du projet démocratique. Et de conclure : «Une réponse est donc nécessaire car, fondées ou non, ces interrogations ont d'ores et déjà des effets bien réels en termes de désenchantement démocratique et d'opposition à l'ouverture»'. Tout cela bien avant le «Brexit» et l'élection de Donald Trump. Parmi les réponses, $P$. Lamy suggère un aménagement des règles de sauvegardes de l'OMC, laissant plus de latitude pour protéger les préférences collectives de chaque pays ou région, le prix à payer - en partie pour démontrer la force de leur attachement à ces préférences - étant de compenser financièrement les partenaires commerciaux éventuellement victimes des sauvegardes en question?2.

\footnotetext{
I. Pascal Lamy, «The emergence of collective preferences in international trade: implications for regulating globalisation», Conférence «Collective preferences and global governance: what future for the multilateral trading system», Bruxelles, 2004. 2. Voir Tristan Le Cotty et Tancrède Voituriez, «Coordination des politiques agricoles et compensations internationales», Les notes de I'IDDRI, n 6, 2006.
} 
Quinze ans plus tard, le débat sur la finalité des ALE se pose dans des termes similaires. Les préoccupations sociétales (emploi, environnement, santé) s'expriment avec une acuité aiguisée par les rapports d'experts établissant une causalité directe entre un certain modèle ou certaines pratiques agricoles et la dégradation d'indicateurs sanitaires et environnementaux'. Dans le même temps, les lecteurs les plus sourcilleux des accords de nouvelle génération expriment leur crainte d'un risque d'omission, d'entrave ou d'étouffement des préférences sociales pris dans son sens le plus large, par un dévoiement des dispositifs contenus par les ALE, au détriment du plus grand nombre et au profit de quelques-uns. C'est le constat que dresse le rapport Schubert en matière d'agriculture dans son analyse du CETA : «Le risque est que le CETA ne fournisse pas des conditions favorables aux objectifs de la transition écologique de l'agriculture (maintien de la place des prairies et de l'association polyculture-élevage notamment), en particulier dans le secteur de l'élevage bovin

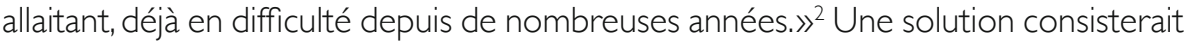
à rétablir l'équilibre des priorités entre multilatéralisme et bilatéralisme, et d'énoncer tout en même temps des objectifs clairs aux ALE et intelligibles par la population. Ceux-ci sont une entorse au multilatéralisme défendu par l'UE et devraient être acceptables et envisagés que s'ils constituent un mieux-disant social et environnemental par rapport au business as usual offert par l'OMC. Cette règle détermine les partenaires avec lesquels I'UE devrait considérer la négociation d'ALE. Règle logique, elle est aussi une règle de pertinence : aucun ALE ne devrait être négocié avec des pays n'ayant signé et ratifié des accords, conventions, traités, jugés fondamentaux par I'UE, comme l'Accord de Paris sur le climat. Et la sortie de l'un d'entre eux suspendrait alors l'application de l'ALE. La France soutient peu ou prou ces principes, les coalitions transpartisanes au nouveau Parlement européen vraisemblablement aussi.

Ainsi les ALE pourraient-ils avant tout servir des objectifs de protection de la santé et de l'environnement - mieux que ne le feraient les accords de l'OMC. Dans un monde où les préférences collectives sont parfois conflictuelles, ces accords «mieux-disant sociaux et environnementaux» permettraient à l'Europe et aux pays partenaires, partageant les mêmes valeurs et intérêts, de peser en étendant, de fait, l'application des normes et standards les plus élevés, conditionnant l'accès à leur marché.

\section{DES POLITIQUES AGRICOLES ET COMMERCIALES EUROPÉENNES AU MILIEU DU GUÉ}

Avec d'impressionnants accomplissements derrière elles, ces politiques sont aujourd'hui critiquées pour les réponses imparfaites qu'elles apportent aux défis

\footnotetext{
I. Xavier Poux et Pierre-Marie Aubert, «Une Europe agroécologique en 2050 : une agriculture multifonctionnelle pour une alimentation saine. Enseignements d'une modélisation du système alimentaire européen 》, IDDR/ Study, n० 9//8, septembre 2018.

2. Jean-Luc Angot et al., op. cit., p. 7.
} 
de nos sociétés. Si les élus et les fonctionnaires de la Commission européenne ne ménagent pas leur peine pour réviser les textes encadrant l'une et l'autre, le risque d'un désarroi croissant à l'égard de deux politiques historiques ressortant aux compétences exclusives de l'UE va grandissant. L'histoire en explique les causes: la PAC s'est depuis 1992, et dans une moindre mesure depuis 2008, réformée par l'impératif indiscutable d'une conformité avec les règles de l'OMC. La chose était contraignante, mais opératoire : elle évitait de réfléchir à ce que l'on voulait faire à la fois pour le commerce, et pour les politiques agricoles. Cette logique opératoire est désormais révolue : l'imminence de négociations à l'OMC ne permet plus de mettre en ordre la PAC, les ALE guère davantage. L'agenda commercial et l'agenda agricole se trouvent, pour la première fois, déliés.

Cette situation inédite est en réalité une opportunité. Opportunité double, qui oblige à se demander ce qui est attendu d'un surcroît de libéralisation commerciale, et à se mettre d'accord sur le sens de la PAC, sans l'excuse ou le prétexte d'un calendrier commercial pour en préciser le trait. Les défis communs posés à ces deux champs de compétence de l'UE sont un précieux guide pour avancer. Pour l'essentiel, ils relèvent des «maux communs » ou «maux publics», au sens économique du terme, et couvrent les aspects non marchands de la production et des échanges - santé et environnement en premier lieu. Les placer au centre des justifications des ALE permettrait à I'UE de continuer de faire du multilatéralisme sa priorité, tout en ménageant un espace confortable pour une réforme de la PAC conforme à ses valeurs et à ses intérêts.

\section{L'ACCORD DE PARTENARIAT ÉCONOMIQUE UE-JAPON (JEFTA, 2017)}

Le 6 juillet 2017, l'Union européenne (UE) et le Japon ont signé un accord de libreéchange (Japan-European Union Free Trade Agreement,JEFTA), point d'aboutissement de trois années d'intenses négociations. La perspective de retrait des États-Unis de l'Accord de partenariat transpacifique (TPP) avait conduit Tokyo à accélérer la phase de négociation afin d'aboutir à un accord avec Bruxelles, dans la perspective notamment d'une préservation des débouchés pour les entreprises japonaises. Le JEFTA fait donc partie de la panoplie des accords qui, depuis de nombreuses années, sont négociés puis signés par l'UE (Ukraine, Chili, Maroc, Canada, Mercosur,AustralieNouvelle-Zélande etc.). L'économie japonaise étant en berne depuis plus d'une décennie, la signature d'accords commerciaux, dont celui réalisé avec l'UE, est perçue comme un levier pour relancer la croissance économique du pays par le truchement des flux commerciaux.

Pour ce qui est du secteur agricole et alimentaire, l'abaissement - ou la suppression des droits de douane consécutif au JEFTA va permettre au Japon de s'approvisionner à moindre coût en produits agricoles et alimentaires, dont il est l'un des tout premiers 
importateurs mondiaux. Le déficit chronique illustre le très faible niveau d'autosuffisance $-40 \%$ seulement - de cette économie de près de 130 millions d'habitants. Le déficit commercial agroalimentaire du Japon avec le reste du monde dépasse assez largement les 50 milliards de dollars en moyenne sur la décennie écoulée - un pic de $7 \mathrm{I}$ milliards ayant été atteint en $20 \mathrm{II}$ '. Le déficit est principalement imputable aux produits de la pêche, aux viandes bovines et de porc, aux céréales et aux fruits et légumes. Il est aussi largement conditionné par la parité du yen vis-à-vis des autres monnaies, plus spécifiquement envers le dollar et l'euro.

L'UE est en effet le deuxième fournisseur du Japon, derrière la Chine et devant les États-Unis, la France n'apparaissant qu'au dixième rang des exportateurs de produits agricoles et alimentaires. Les producteurs français ont ainsi vu dans le JEFTA une opportunité pour conquérir des parts de marché au Japon, en particulier dans le secteur de la viande bovine, de la charcuterie, du foie gras - le Japon en constitue le premier importateur mondial, malgré la suspension des achats lors de l'épisode d'influenza aviaire en 2016 -, des vins et alcools et des produits laitiers. En contrepartie d'un approvisionnement plus optimal en provenance de l'UE, le Japon va, pour sa part, pouvoir mieux pénétrer les marchés des 27 États membres en biens issus des industries de l'électronique et de l'automobile.

En signant ce type d'accord avec l'UE, le Japon fait toutefois prendre un tournant à ses agriculteurs. L'une des ambitions des autorités japonaises est en effet de les faire entrer dans un système d'économie ouverte, alors qu'ils ont été historiquement protégés par un syndicat professionnel - le JA-Zenchu - extrêmement puissant et offensif. L'agriculture japonaise est l'une des plus protégées et soutenues du monde. Alors que des accords commerciaux signés avec le Canada (CETA), négociés avec l'Australie et la Nouvelle-Zélande, ou avec le Mercosur (Argentine, Paraguay, Uruguay et Brésil), ont soulevé des polémiques, liées notamment aux différences dans les conditions de production des produits et au risque que pourraient courir des filières européennes comme celle de la viande bovine, le JEFTA a été, somme toute, bien accepté dans les pays de l'UE, en raison des intérêts offensifs et des gains économiques qu'il procurerait aux producteurs européens.

Thierry Pouch

Chef du Service études, références et prospective des Chambres d'agriculture de France, chercheur associé au Laboratoire REGARDS de I'Université de Reims

Champagne-Ardenne et membre de l'Académie d'agriculture de France

I. Source : CEPII - CHELEM. 


\section{FROM DEFENSE TO ATTACK: THE CASE OF THE EU GEOGRAPHICAL INDICATIONS SYSTEM}

In 1992, at dawn of the late-20th-century era of global trade liberalisation, the European Community (EC, the "European Union" name was yet to come) established a sui generis intellectual property scheme to protect food products whose characteristics are attributable essentially to their place of origin. These products, known as Gls (Geographical Indications) in the global trade jargon, are nothing new. There are more than 10,000 protected Gls around the world, in a "mosaic" of registers and protection systems. In the first decades of the 20th century already, France established laws to protect $\mathrm{GI}$ of wines and cheeses, followed by Italy and Spain. The EC itself has had legislation in place for wines' Gl since the 1970s. The 1994 World Trade Organisation (WTO) Agreement on Trade-Related Aspects of Intellectual Property Rights (TRIPS) provides a common definition of Gls recommending the creation of a "multilateral system of notification and registration for geographical indications for wines", establishing de facto a higher level of protection for wines and spirits than for foodstuffs.

Against this background, the 1992 regulation is a remarkable step because of its timing and the peculiarity of the EU system. In fact, Europe started to create a system linking the quality of food to its production place, while in the same years the new global trade rules set standards relying on the "material" characteristics of food only, not including origin, considered to be an "immaterial" element. Thus, from its inception, the EU GI system stands on the frontier of the 1990s global trade order.

In the EU Gl scheme, the place of provenance is the driver of the supply system configuration, going far beyond a simple origin labelling. The main goal is protecting and encouraging the diversification of agricultural production in order to contribute to the development of rural territories and keeping vitality in less-favoured areas. More interestingly, a second aim is to keep the pace with the evolution of food demand, that was, at that time in Europe, already giving an increasing value to immaterial elements of food, such as taste, uniqueness and cultural features. In the attempt of grasping the change in food consumption trends in the internal market, the European institutions, maybe unintentionally, anticipated the tastes of the raising global middleclass, in which an increasing wealth is associated with more sophisticated food consumption patterns, relying on distinctiveness and attractiveness of premium and "niche" products.

Thus, what was originally conceived as a defensive tool has become an offensive interest in every trade negotiation undertaken by the EU worldwide. South Korea, Canada, Japan, Singapore, Peru, Colombia, Vietnam, Mexico, etc. In all these markets, thanks to bilateral trade agreements, the EU achieved to promote the Gls as a European golden standard, obtaining a high-level protection for EU specialities names. The result is the connection of traditional skills and local know-hows to the 
international markets. The EU Protected Designation of Origin (PDO) and Protected Geographical Indication (PGI) logos - accorded only to farmers and cooperatives respecting specific and costlier-than-the-norm production requirements - may be granted as a collective brand, enabling thousands of small farms and cooperatives to participate in the global economy, promoting efficiency and equity, and contributing to maintain the diversity, cultural identity and economic vitality of local food systems and territories.

Today more than 3,870 European Gls are protected in the EU, including over I,940 wines, I,650 agricultural products and foodstuffs, 270 spirits and 5 aromatised wine products. More importantly, "Gls represent some $€ 55$ billion of sale value and more than $15 \%$ of the total Union food and drink export"', writes oriGln Europe (Organisation for an International Geographical Indications Network), the European branch of oriGln, the global alliance of Gl producers.

Yet, the EU approach to Gl protection is still one of the most controversial issues in global trade, especially in transatlantic relations. The economist Tim Josling called it "The War on Terroir"2. For the United States (US) trade administration, Gls as intended in Europe are pure protectionism. The US has always opposed the recognition of a reinforced legal protection to $\mathrm{Gl}$ food and has never fully implemented the TRIPS agreement on wine.

The EU-Canada Comprehensive Economic and Trade Agreement (CETA), partially entered into force in 2017, has been a turning point. Due to the CETA, Canada - that had always sided with the US against the EU Gls system - has recognised a list of hundreds Gls and changed its domestic regulation on trademarks, establishing a register, allowing the high level of protection for $\mathrm{Gl}$ foods beyond wines and spirits. Another traditional US ally, Australia, is also negotiating a trade agreement including Gls with the EU.

At dusk of the WTO era in global trade, the EU definition of Gls, relying on terroir only, might need some update. This is the idea of an increasing number of $\mathrm{Gl}$ consortia, especially those able to export in global markets. Producers want to expand the concept of food quality recognised by the EU law, adding environmental, social, economic and ethical (animal welfare, fair remuneration of farmers) aspects to the traditional definition based on territory, history and savoir-faire.

\section{Angelo Di Mambro}

Environmental and agri-food journalist

\footnotetext{
I. oriGln, «Geographical Indications - an asset for the European Union. oriGln EU proposals to reinforce the European Quality Policy», 16 May 2019.

2. Johan Swinnen and Martijn Huysmans, «No Terroir in the Cold? A Note on the Geography of Geographical Indications», Journal of Agricultural Economics, n 70, April 2019.
} 